\title{
The Effect of Carrier Phase on GPS Multipath Tracking Error
}

\author{
Omer Mohsin Mubarak \\ Department of Electrical Engineering \\ Jouf University \\ Saudi Arabia \\ ommubarak@ju.edu.sa
}

\begin{abstract}
Multipath is one of the main sources of tracking error in GPS receivers. This tracking error has previously been analyzed against the relative delay of the Line of Sight (LOS) and reflected signals. However, only carrier phase differences of 0 and $\pi$ were used, since they give tracking error with maximum magnitude. This paper shows that tracking error does not change linearly with changing carrier phase difference. Tracking error plots against relative carrier phase difference of the LOS and reflected signals have been used to analyze the relationship between the two in various scenarios. While maximum positive and negative errors are found at carrier phase difference of 0 and $\pi$, a sharp increase in tracking error is found around the phase difference of $\pi$. There is a zero crossing in all plots but that point is dependent on relative amplitude, delay, and carrier phase difference of the two signals. The analysis has also been extended to narrow correlators receiver. Tracking error is significantly reduced in this case, however, similar characteristics have been observed when the tracking error is analyzed against the relative carrier phase difference. Moreover, the tracking error was found to be less dependent on the relative delay between the two signals when correlators spacing is reduced.
\end{abstract}

Keywords-multipath; global navigation satellite system; global positioning system; carrier phase; tracking error

\section{INTRODUCTION}

The reflection of the Line of Sight (LOS) satellite signal from nearby objects, also known as multipath, causes tracking errors in the Global Positioning System (GPS) receivers [1-3]. Tracking errors caused by multipath can be positive or negative and depend on relative amplitude, carrier phase, and code delay of the reflected signal with respect to the LOS signal [4]. A plot of maximum positive and maximum negative code tracking errors against relative delay of a reflected signal is called as the multipath error envelope and is generally used to represent error characteristics [5-7] and effects of mitigation techniques [8-9]. It shows the maximum deviation in tracking error that can be caused by variations of carrier phase difference between the LOS and the reflected signals for a given relative amplitude and code delay between the two signals. Two maximums are obtained for relative carrier phase differences of 0 and $\pi$ radians. However, the error envelope does not provide details of how tracking error is changed when the carrier phase difference between the two signals changes from 0 to $\pi$ or vice versa. This paper analyzes the effect of carrier phase difference between the LOS and the reflected signals on multipath tracking error.

\section{EXPERIMENTAL SETUP}

In a GPS receiver, correlators are placed on the correlation function to track the code phase of a received GPS signal. Typically, three correlators are used, one at the prompt or on time correlation position and other two (early and late) symmetrically placed on either side [7, 10]. Early and late correlators use equally advanced and delayed versions of prompt code respectively, such that for a triangular correlation function their equal energy implies that prompt correlator tracks the peak. Equal energy is ensured using a discriminator function, which in simplest form is the difference in energy of the two. Tracking loops adjust local code and carrier, aiming to maintain discriminator output to zero. In the presence of a reflected signal, the correlation function shape is distorted. In this case, even with zero output of discriminator function, prompt correlator code is not aligned with received signal, resulting in tracking error [2]. In this paper, a perfect triangular function $v(t)$ is used as an autocorrelation function for determining tracking error. The counterfeit signal is given as scaled, phased and delayed version of $v(t)$. The received signal $g(t)$ is then given as the sum of the LOS and counterfeit signal by:

$$
g(t)=v(t)+\alpha e^{j \varphi} v(t-\tau)
$$

where $\alpha, \varphi$ and $\tau$ are respectively the relative amplitude, the carrier phase difference, and the delay of the counterfeit signal with respect to LOS. The discriminator function is given by:

$$
e(t)=D_{l}(t) D_{l}^{*}(t)-D_{e}(t) D_{e}^{*}(t)
$$

where $D_{l}$ and $D_{e}$ are given by (3) and (4) respectively.

$$
\begin{aligned}
& D_{l}(t)=v(t+\epsilon)+\alpha e^{j \varphi} v(t+\epsilon-\tau) \\
& D_{e}(t)=v(t-\epsilon)+\alpha e^{j \varphi} v(t-\epsilon-\tau)
\end{aligned}
$$

where $\epsilon$ is the correlator spacing between early and prompt, or prompt and late correlators. Wide correlators, i.e. with 1 chip spacing between early and late correlators ( $\epsilon=0.5$ chips), are used in this paper, except in Section V. 


\section{MotIVATION}

Figure 1 shows the tracking error for a multipath signal with relative amplitude of 0.5 and carrier phase offsets $(\varphi)$ of 0 , $\pi / 4, \pi / 2,3 \pi / 4$, and $\pi$, with respect to the LOS signal. Relative amplitude of 0.5 implies that reflected signal is of half amplitude than the LOS signal and $\varphi=0$ implies that the reflected signal is in-phase with the LOS signal. The tracking error is zero in all cases for reflected signal delay of over 1.5 chips. Plots confirm that maximum error magnitude is obtained when the reflected signal is in-phase or completely out of phase $(\varphi=\pi)$ with the LOS signal. However, it can also be noted that plots are not uniformly spaced as phase difference is increased by $\pi / 4$. For example, plots for $\varphi=0$ and $\varphi=\pi / 4$ are much closer as compared to the plots for $\varphi=\pi / 4$ and $\varphi=\pi / 2$, although in both cases the difference in phase is $\pi / 4$.

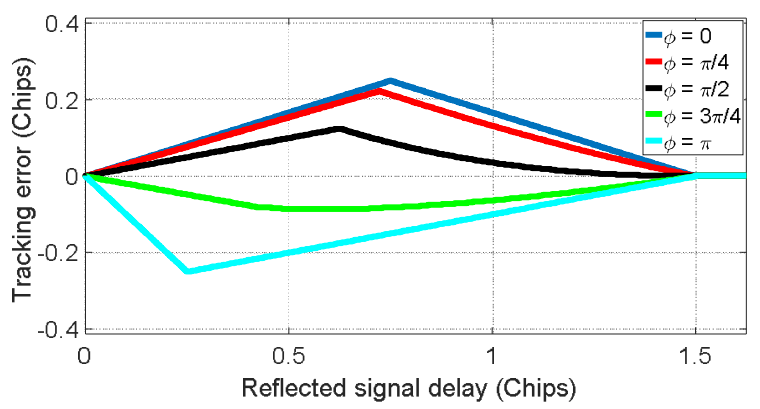

Fig. 1. Tracking error plot against signal's relative delay for a multipath signal with relative amplitude of 0.5 and varying carrier phase offsets $(\varphi)$ with respect to the LOS signal.

Similar patterns are observed for other relative amplitudes of multipath signal with respect to the LOS signal. Figures 2 and 3 show the tracking errors for a multipath signal with relative amplitude of 0.3 and 0.8 respectively. It can be observed that the overall error magnitude is smaller for relative amplitude of 0.3 and higher for relative amplitude of 0.8 , as compared to Figure 1. However, similar to Figure 1, the plots in both figures are not uniformly spaced as phase difference is increased by $\pi / 4$.

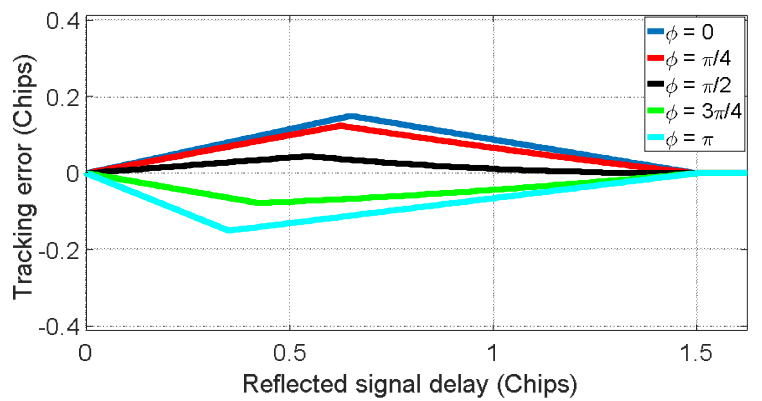

Fig. 2. Tracking error plot against signal's relative delay for a multipath signal with relative amplitude of 0.3 and varying carrier phase offsets $(\varphi)$ with respect to the LOS signal.

Non-linear change in tracking error with changing carrier phase difference provided motivation to explore the effect of phase difference between the LOS and reflected signals on tracking error.

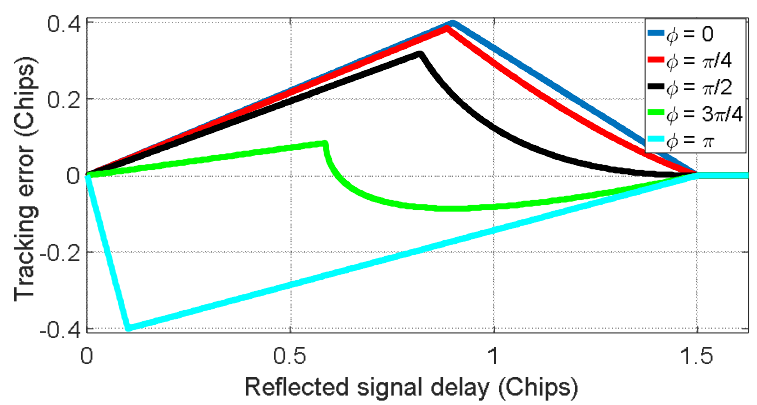

Fig. 3. Tracking error plot against signal's relative delay for a multipath signal with relative amplitude of 0.8 and varying carrier phase offsets $(\varphi)$ with respect to the LOS signal.

\section{TRACKING ERROR ANALYSIS}

This section analyzes the changes in tracking error with changing carrier phase difference between the LOS and reflected signals. Figures 4-6 show the tracking error plots against relative carrier phase difference of the LOS and reflected signals, with relative amplitudes of $0.3,0.5$, and 0.8 . respectively.

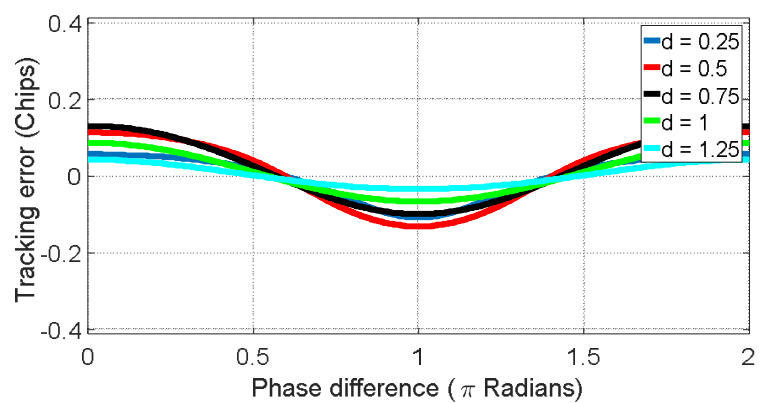

Fig. 4. Tracking error plot against signal's relative phase difference for a multipath signal with relative amplitude of 0.3 and varying multipath signal delay $(d)$ with respect to the LOS signal.

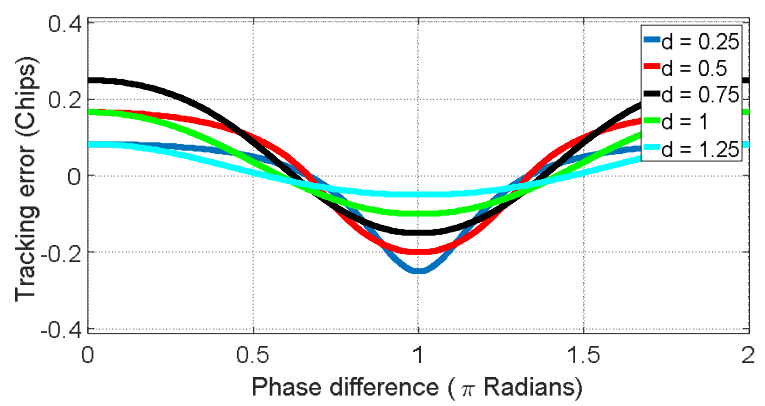

Fig. 5. Tracking error plot against signal's relative phase difference for a multipath signal with relative amplitude of 0.5 and varying multipath signal delay $(d)$ with respect to the LOS signal.

The following can be observed from these plots:

- It is confirmed that for a given relative amplitude and delay between signals, the maximum positive error is obtained for $\varphi=0$ and the maximum negative error is obtained for $\varphi=\pi$, in all cases. 
- The change in tracking error with increasing carrier phase difference is non-linear for all cases.

- There is a sharp increase in tracking error around $\varphi=\pi$ for all cases.

- There is no single phase difference which can give zero tracking error in all cases, since zero crossing for each plot is different. Zero crossing of a plot is dependent on all three parameters, i.e. relative amplitude, delay, and carrier phase difference between the LOS and reflected signals.

- Figure 4 plots have the least variation in tracking error as phase difference is changed from 0 to $\pi$ radians. This implies that the dependence of the tracking error on carrier phase difference is higher for higher relative amplitudes of the reflected signal.

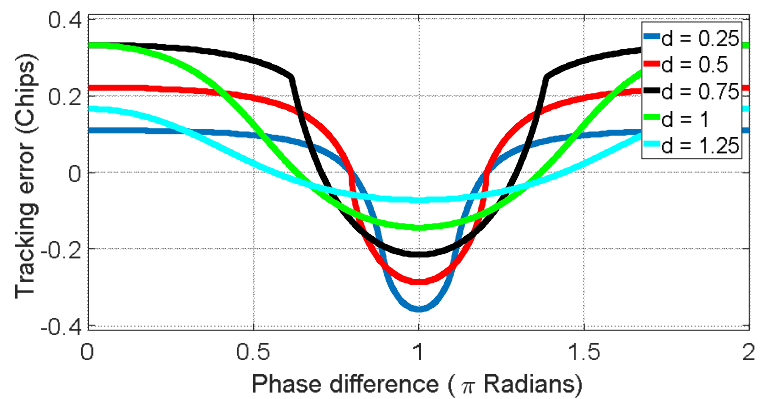

Fig. 6. Tracking error plot against signal's relative phase difference for a multipath signal with relative amplitude of 0.8 and varying multipath signal delay $(d)$ with respect to the LOS signal.

\section{TRACKING ERROR WITH NARROW CORRELATOR}

The previous analysis has used wide correlators, i.e. 1 chip spacing between early and late correlator of a receiver. Reduced spacing of 0.1 chip between early and late correlators, termed as the narrow correlator, has been used for mitigating the tracking error caused by multipath for various global navigation satellite signals $[8,11-13]$. This can be confirmed from Figure 7, which shows the tracking error with reflected signal relative amplitude of 0.5 using a narrow correlator.

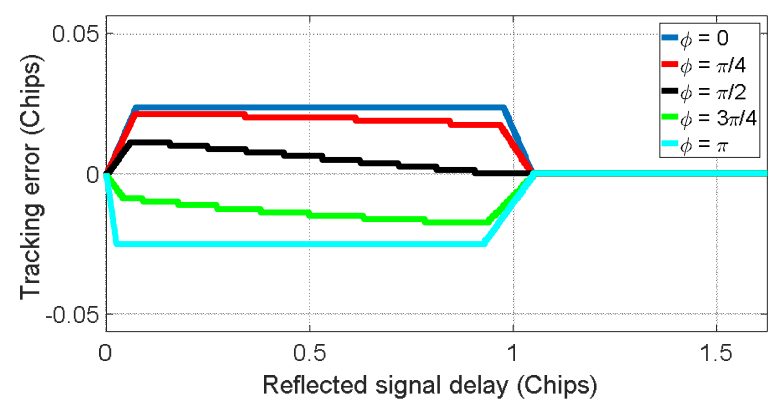

Fig. 7. Tracking error plot against signal's relative delay for a multipath signal with relative amplitude of 0.5 and varying carrier phase offsets $(\varphi)$ with respect to the LOS signal, using narrow correlators in receiver.

Comparing this with Figure 1, it can be seen that the tracking error has been significantly reduced. The tracking error is zero when separation between the LOS and reflected signals is more than 1.05 chips, instead of 1.5 chips in Figure 1. The maximum tracking error is 0.025 chips instead of 0.25 chips obtained with wide correlators. Similarly, reduced error can be observed in Figures 8-9, as compared with similar relative amplitude plots in Figures 2-3 respectively. It can again be noted that the plots in Figures 7-9 are not uniformly spaced. Similar to wide correlators, plots for $\varphi=0$ and $\varphi=\pi / 4$ are much closer than the plots for $\varphi=\pi / 4$ and $\varphi=\pi / 2$, although in both cases the difference in phase is $\pi / 4$. Therefore, the change in tracking error against carrier phase difference is analysed in this section for a narrow correlator receiver.

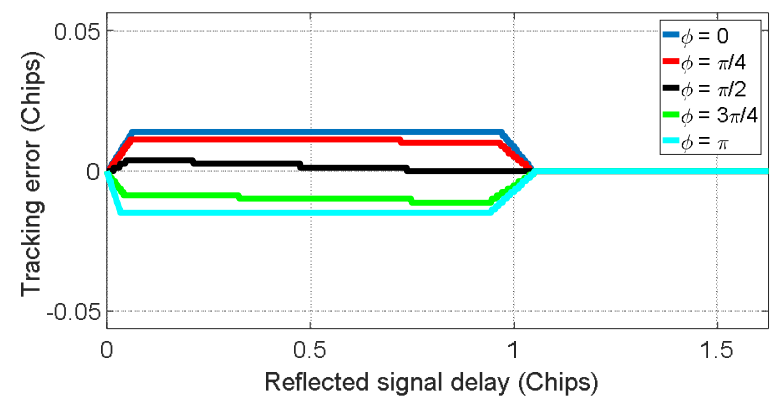

Fig. 8. Tracking error plot against signal's relative delay for a multipath signal with relative amplitude of 0.3 and varying carrier phase offsets $(\varphi)$ with respect to the LOS signal, using narrow correlators in receiver.

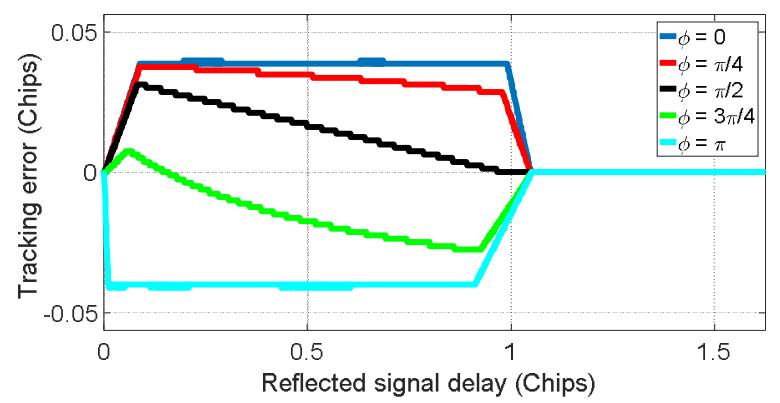

Fig. 9. Tracking error plot against signal's relative delay for a multipath signal with relative amplitude of 0.8 and varying carrier phase offsets $(\varphi)$ with respect to the LOS signal, using narrow correlators in receiver.

Figures 10-12 show tracking error plots against relative carrier phase difference of the LOS and reflected signals using narrow correlators in a receiver, with relative amplitudes of 0.3 , 0.5 , and 0.8 respectively. All the 5 observations noted in the previous section for wide correlators, are also valid for these narrow correlator based plots. Moreover, out of delays of 0.25 , $0.5,0.75,1$, and 1.25 chips, the 1.25 chips plot stays at zero in all the 3 Figures, as the reflected signal delay of over 1.05 chips gives zero tracking error irrespective of the relative amplitude and carrier phase difference of the reflected signal with respect to the LOS signal. It can also be noted that plots are much closer as compared to the plots obtained using wide correlators for the same relative amplitude. For example, the plots in Figure 10 are much closely spaced as compared to the plots in Figure 4, although both are obtained for relative amplitude of 0.3 and the same set of relative delays. 


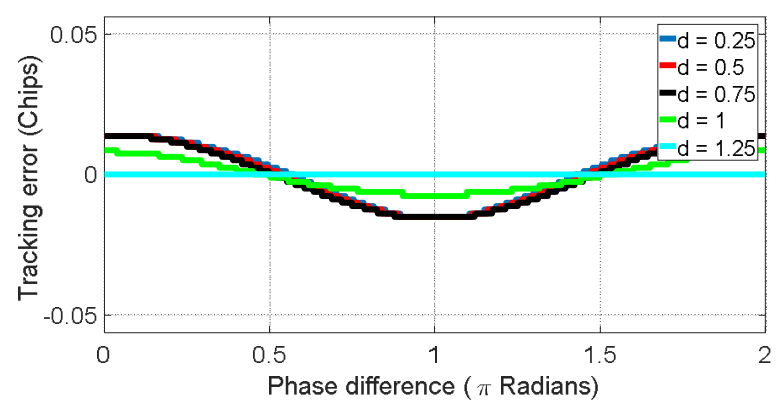

Fig. 10. Tracking error plot against signal's relative phase difference for a multipath signal with relative amplitude of 0.3 and varying multipath signal delay (d) with respect to the LOS signal, using narrow correlators in receiver.

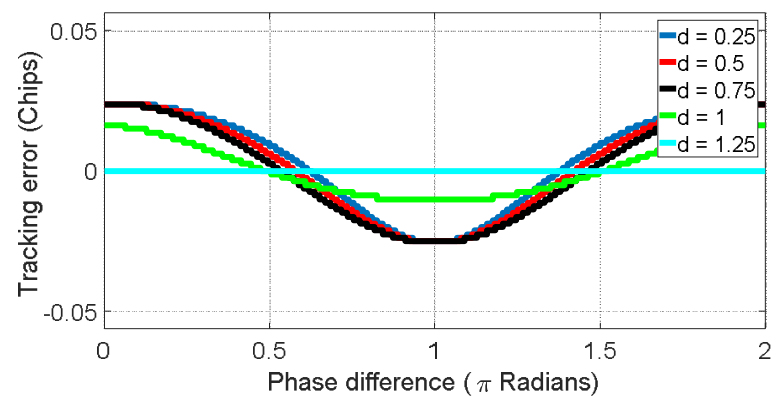

Fig. 11. Tracking error plot against signal's relative phase difference for a multipath signal with relative amplitude of 0.5 and varying multipath signal delay (d) with respect to the LOS signal, using narrow correlators in receiver.

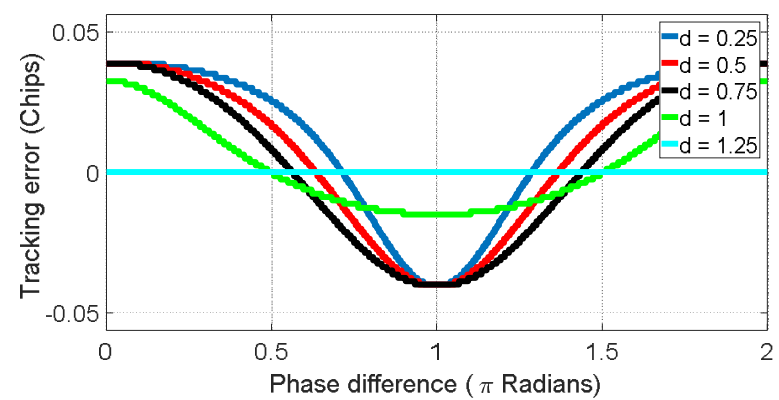

Fig. 12. Tracking error plot against signal's relative phase difference for a multipath signal with relative amplitude of 0.8 and varying multipath signal delay (d) with respect to the LOS signal, using narrow correlators in receiver.

\section{CONCLUSION}

Multipath is a source of tracking error in GPS receivers, which leads to positioning errors [3, 14-17]. Multipath error envelopes have been used to analyze tracking error caused by multipath [5-9]. However, they only provide maximum positive and negative error for a given relative amplitude and code delay between the two signals. Two maximums are obtained for relative carrier phase differences of 0 and $\pi$ radians, whereas the tracking error for relative carrier phase differences between 0 and $\pi$ radians has not been explored earlier. This paper has analyzed the tracking error caused by multipath and specifically the effect of carrier phase on error. Tracking error plots against relative carrier phase difference of the LOS and reflected signals have been used instead of conventional tracking error plots against relative delay of the two signals. This novel analysis confirmed that maximum positive and negative errors are obtained for $\varphi=0$ and $\varphi=\pi$ respectively. It has also been observed that change in tracking error with increasing carrier phase difference is non-linear and the error increases sharply around $\varphi=\pi$. The zero crossing of a tracking error plot is found to be dependent on relative amplitude, delay and carrier phase difference between the LOS and the reflected signals, i.e. there is no single carrier phase difference between the two signals which gives zero tracking error. Moreover, the dependence of tracking error on carrier phase difference is found to be higher for higher relative amplitude of the reflected signal.

Tracking error has also been analyzed for a receiver using narrow correlators, which are generally used to reduce the tracking error caused by multipath. The spacing between early and late correlators was reduced to 0.1 chip, instead of 1 chip in wide correlators. As a result, maximum tracking error reduced to 0.025 chips instead of 0.25 chips. Moreover, tracking error is zero when the separation between the LOS and reflected signals is more than 1.05 chips, instead of 1.5 chips for wide correlator receivers. Characteristics observed for tracking error using wide correlators are also found to be valid when narrow correlators were used. Moreover, the plots for different multipath signal delays are found to be much closer as compared to the plots obtained using wide correlators for the same set of signal parameters. This implies that the tracking error is less dependent on relative delay between the two signals when narrow correlators are used.

These findings can be useful for finding better estimates of tracking error in a GPS receiver for multipath with given relative amplitude, carrier phase difference, and delay between the two signals.

\section{REFERENCES}

[1] X. Chen and F. Dovis, "Enhanced CADLL Structure for Multipath Mitigation in Urban Scenarios," in Proceedings of the 2011 International Technical Meeting of The Institute of Navigation, San Diego, CA, 2011, pp. 678-686.

[2] O. M. Mubarak and A. Dempster, "Carrier phase analysis to mitigate multipath effect," presented at the IGNSS Symposium 2007, The University of New South Wales, Sydney, Australia, Dec. 2007.

[3] O. M. Mubarak and A. G. Dempster, "Exclusion of Multipath-Affected Satellites Using Early Late Phase," Journal of Global Positioning Systems, vol. 9, no. 2, pp. 145-155, 2010.

[4] K. Yedukondalu, A. D. Sarma, and V. S. Srinivas, "Estimation and mitigation of GPS multipath interference using adaptive filtering," Progress In Electromagnetics Research M, vol. 21, pp. 133-148, 2011, doi: 10.2528/PIERM11080811.

[5] T. G. Ferreira and F. D. Nunes, "Advanced multipath mitigation techniques for GNSS receivers," presented at the 1st Seminar of the Portuguese Committee, Lisbon, Portugal, Nov. 2007.

[6] O. M. Mubarak and A. G. Dempster, "Analysis of early late phase in single-and dual-frequency GPS receivers for multipath detection," GPS Solutions, vol. 14, no. 4, pp. 381-388, Sep. 2010, doi: 10.1007/s10291010-0162-z.

[7] A. Pirsiavash, A. Broumandan, and G. Lachapelle, "Characterization of Signal Quality Monitoring Techniques for Multipath Detection in GNSS Applications," Sensors (Basel, Switzerland), vol. 17, no. 7, Jul. 2017, doi: $10.3390 / \mathrm{s} 17071579$. 
[8] A. J. V. Dierendonck, P. Fenton, and T. Ford, "Theory and Performance of Narrow Correlator Spacing in a GPS Receiver," NAVIGATION, vol. 39, no. 3, pp. 265-283, 1992, doi: 10.1002/j.2161-4296.1992.tb02276.x.

[9] A. Pirsiavash, A. Broumandan, and G. Lachapelle, "Performance Evaluation of Signal Quality Monitoring Techniques for GNSS Multipath Detection and Mitigation," presented at the International Technical Symposium on Navigation and Timing (ITSNT), Toulouse, France, Nov. 2017.

[10] E. D. Kaplan, Understanding GPS: Principles and Applications, Second Edition, 2nd edition. BS: Artech House, 2005.

[11] M. E. Cannon, G. Lachapelle, W. Qiu, S. L. Frodge, and B. Remondi, "Performance analysis of a narrow correlator spacing receiver for precise static GPS positioning," in Proceedings of 1994 IEEE Position, Location and Navigation Symposium - PLANS'94, Apr. 1994, pp. 355-360, doi: 10.1109/PLANS.1994.303337.

[12] Z. Xuefen, C. Xiyuan, and C. Xin, "Comparison between strobe correlator and narrow correlator on MBOC DLL tracking loop," in 2011 IEEE International Instrumentation and Measurement Technology Conference, May 2011, pp. 1-4, doi: 10.1109/IMTC.2011.5944083.

[13] J. H. Lee et al., "A GPS multipath mitigation technique using correlators with variable chip spacing," E3S Web of Conferences, vol. 94, 2019, doi: 10.1051/e3sconf/20199403006, Art. No. 03006.

[14] M. Orabi, J. Khalife, A. A. Abdallah, Z. M. Kassas, and S. S. Saab, “A Machine Learning Approach for GPS Code Phase Estimation in Multipath Environments," in 2020 IEEE/ION Position, Location and Navigation Symposium (PLANS), Apr. 2020, pp. 1224-1229, doi: 10.1109/PLANS46316.2020.9110155.

[15] T. Kos, I. Markezic, and J. Pokrajcic, "Effects of multipath reception on GPS positioning performance," presented at the ELMAR-2010, Zadar, Croatia, Sep. 2010.

[16] I. Rumora, N. Sikirica, and R. Filjar, "An Experimental Identification of Multipath Effect in GPS Positioning Error," TransNav, International Journal on Marine Navigation and Safety od Sea Transportation, vol. 12, no. 1, pp. 29-32, Mar. 2018, doi: 10.12716/1001.12.01.02.

[17] T. L. Dammalage, "The Effect of Multipath on Single Frequency C/A Code Based GPS Positioning," Engineering, Technology \& Applied Science Research, vol. 8, no. 4, pp. 3270-3275, Aug. 2018.

\section{AUTHOR'S PROFILE}

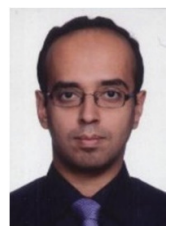

Omer Mohsin Mubarak received the B.S. in Electronics Engineering from the Ghulam Ishaq Khan Institute of Engineering Sciences \& Technology, Pakistan, and the M.E. and Ph.D. degrees from the University of New South Wales, Australia in 2006 and 2010 respectively. From 2013 to 2016, he was with Iqra University, Pakistan and during this period he served as Head of the Electronics Engineering department and Head of Computing \& Technology department. He is currently working as an Assistant Professor at Jouf University, Saudi Arabia. His research interests include multipath mitigation, spoofing detection and other signal processing techniques for GNSS receivers. He is a senior member of IEEE, USA. 